\title{
Implementation of portable common building ground resistance measuring instrument
}

\author{
ChaoLu ${ }^{1}$, Zhiqiang $\mathrm{Chen}^{1}$, XiuShan $\mathrm{Wu}^{1}$, Zhikai Zhao ${ }^{1}$, Hui Peng ${ }^{2}$ \\ ${ }^{1}$ College of Mechanical and Electrical Engineering, China Jiliang University, Hangzhou, Zhejiang, \\ China \\ ${ }^{2}$ Kashi Normal University, Kashi, Xinjiang, China
}

Keywords: ground resistance; three-pole compensation method; FFT; STM32

\begin{abstract}
According to the defect of the building ground resistance measuring device presently. A new system of building ground resistance detection which based on STM32 was proposed. This scheme was based on the principle of three-pole compensation method. Hardware and software of the master-slave machine which controlled by embedded system was applied to achieve accurate measurement of ground resistance. The detection system included synthesizer of alternating current, signal collecting and data processing platform. The detection system was consisted of a STM32 control which product alternating current with high precision frequency and waveform stability into the grounding grid. The signal of voltage and current in the grounding grid were translated into a precise grounding resistance value through band-pass filter, differential amplify, synchronous AD conversion, FFT processing. The detection system could improve the detection accuracy and efficiency, and realize the intelligent testing work.
\end{abstract}

\section{Instruction}

Building grounding device plays an important role in the whole building lightning protection and normal operation of electrical equipment. Grounding resistance is one of the main technical parameters of grounding device, and an important index to measure the quality of the lightning protection engineering $^{[7]}$. So the accurate detection of grounding resistance is important to ensure the safe operation of electrical equipment in buildings and personal safety. With the rapid development of modern cities, buildings especially high-rise buildings concentrated in cities, which puts forward a more difficult requirement for building grounding resistance test. The China meteorological administration currently uses mainly shake table style and 4105A-type ground resistance detector ${ }^{[3]}$. Such instruments were operated more cumbersome, and the digital level is not high, the accuracy needs to be improved. This paper selects enhanced STM32 chip to build detection system, and uses 12 bit high-speed parallel AD converter chip AD7656 and highperformance signal conditioning circuit to achieve accurate detection of ground resistance.

\section{Detection Principle}

When current diffuses from earth electrode to surrounding land, soil will be showing resistance characteristics, which is called ground resistance. According to ohm's law in ground resistance measurement, the current and voltage that flow through ground pole should be got firstly, then the formula $R=U$ / I will be used to get the ground resistance value. In the actual detection, in order to simplify the calculations, grounding grid was regarded as hemisphere, as shown in figure 1 . When measuring, current probe should be placed at zero potential to eliminate the influence of grounding. However in actual wiring, long distance wiring is not possible. Three-pole compensation method was usually used in the grounding resistance detection, and it's principle is shown in Figure $1^{[1][5][6]}$. Using electric field intensity formula $E=J \rho$ and potential equation $U=\int_{\infty}^{x}-E d t$ get the potential which distance from center of sphere of $r$. 


$$
U_{r}=\int_{\infty}^{x}-E d t=\int_{\infty}^{x}-\frac{\rho I}{2 \pi r^{2}} d t
$$

The potential difference between earth electrode and voltage pole can be got by the formula 1 . and the potential difference $U_{C E P}$ between earth electrode and voltage pole which is due to the presence of the current pole also can be got.

$$
\begin{aligned}
& U_{E P}=\frac{\rho I}{2 \pi}\left(\frac{1}{r_{0}}-\frac{1}{d_{1}}\right) \\
& U_{C E P}=\frac{\rho I}{2 \pi}\left(\frac{1}{d_{2}}-\frac{1}{d_{3}}\right)
\end{aligned}
$$

Then get the total potential between earth electrode and voltage pole.

$$
U=U_{E P}+U_{C E P}=\frac{\rho I}{2 \pi}\left(\frac{1}{r_{0}}-\frac{1}{d_{1}}+\frac{1}{d_{2}}-\frac{1}{d_{3}}\right)
$$

According to the resistance formula $R=U / I$, grounding resistance value can be calculated in this wiring method.

$$
R=\frac{U}{I}=\frac{\rho}{2 \pi}\left(\frac{1}{r_{0}}-\frac{1}{d_{1}}+\frac{1}{d_{2}}-\frac{1}{d_{3}}\right)
$$

And the actual spherical earth electrode of resistance can be expressed as

$$
R_{0}=\frac{\rho}{2 \pi r_{0}}
$$

In order to make the actual detection of the grounding resistance value and the theoretical value of ground resistance equal, the formula 5 and formula 6 must be equal, and then get formula 7 .

$$
\frac{1}{r_{0}}-\frac{1}{d_{1}}+\frac{1}{d_{2}}-\frac{1}{d_{3}}=0
$$

Let $d_{1}=\alpha d_{3}, d_{2}=(1-\alpha) d_{3}$, formula 8 can be got.

$$
\frac{1}{1-\alpha}-\frac{1}{\alpha}-1=0
$$

$\alpha$ can be obtained by the above formula is equal to 0.618 . In other words, when the current pole can be wired at infinity, Voltage pole can be put away from earth electrode of $0.61 d_{3}$. This method can also be measured the real grounding resistance. To reduce the mutual inductance between the electrodes, here $d_{3}$ must take proper spacing. Generally $d_{3}$ takes maximum diagonal of grounding grid of $4 \sim 5$ times. Through the above analysis, three-pole compensation method can be used for wiring work of grounding resistance detection. For many interference signals appearing during detection, Such as grounding stray current, high frequency interference and frequency interference etc. hardware and software denoising need be adopted to filter the interference and the measurement accuracy can be improved.

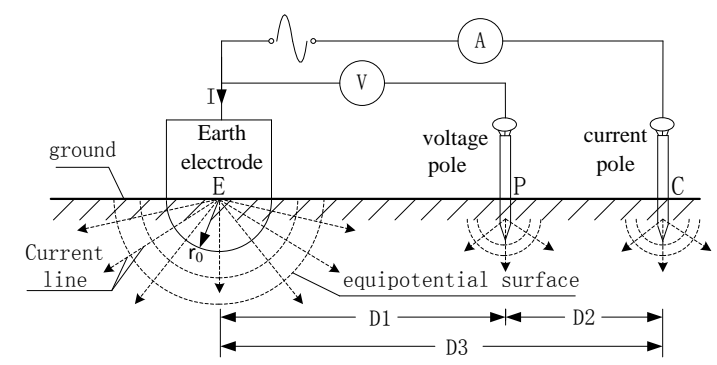

Fig1 Principle diagram of grounding resistance detection 


\section{Hardware design}

The main components of the building ground resistance system as shown in Figure 2. The hardware part of the detecting device is mainly composed of ARM platform which carries the WinCE system, alternating current synthesis module, voltage and current collecting part and peripheral circuit. System uses embedded ARM platform that has the function of human-computer interaction, data processing and storage, control command sending, status display. Alternating current synthesis module that is controlled by STM32 generates sinusoidal current, which is used to inject into the grounding grid. In the collection of voltage and current, signal through the RC filter, differential amplifier, the two order band-pass filtering, AD transformation, FFT processing to obtain the useful signal.

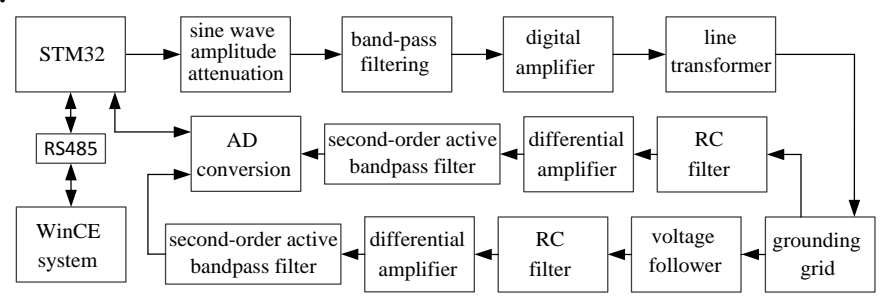

Fig2 Structure diagram of grounding resistance measuring system

\section{Alternating synthesis}

As shown in figure 2, in the AC synthesis module, waveform generating circuit generates sine wave signal which through the digital power amplifier and the line transformer can get the required alternating current signal. Waveform generating circuit uses LM285 to generate a stable reference voltage for DAC. DAC7513 generates sine wave signal which is controlled by STM32, after bandpass filtering, stable sinusoidal current can be got. Because of the existence of geoelectric capacitive reactance $^{[2]}$, The working frequency of the selected AC current must be coupled with geoelectric capacitive reactance, otherwise the influence of geoelectric capacitive reactance can't be reduced. This design uses $800 \mathrm{~Hz}$ which is commonly used in building grounding resistance detection as the working frequency of the alternating current, in order to eliminate the effect of power frequency interference and geoelectric capacitive reactance ${ }^{[7]}$.

\section{Voltage and current collecting and processing}

\section{1) Signal conditioning circuit}

When voltage and current signal collecting, the signal is easily disturbed by electromagnetic signals from the outside world and noise generated by the amplifier circuit itself. In order to obtain the accurate values of the voltage and current, signals need to be conditioned. As shown in Figure 3, in the current signal conditioning circuit, the current signal uses differential input, which through the piezoresistor that can prevent the instantaneous voltage mutation which may damage the circuit. Then we use the sampling resistance to collect current, signal through the low pass filter composed of capacitorsC1C2, clamping voltage protection circuit composed of 9013 type transistors Q1Q2, second-order active band-pass filter can get effective current signal $I_{\text {outo }}$ But in order to ensure the effective signal collection, the signal should be amplified to $I_{\text {out } 1}$. To ensure that current does not decay, the voltage follower is added before amplifier circuit. Two current signals $I_{\text {out }} I_{\text {out } 1}$ are used to improve the precision of $\mathrm{AD}$ conversion. The conditioning circuit of voltage signal, on the whole, as well as current circuit, only string into larger partial pressure resistance before sampling resistance can realize the voltage signal conditioning. 


\section{2) AD conversion}

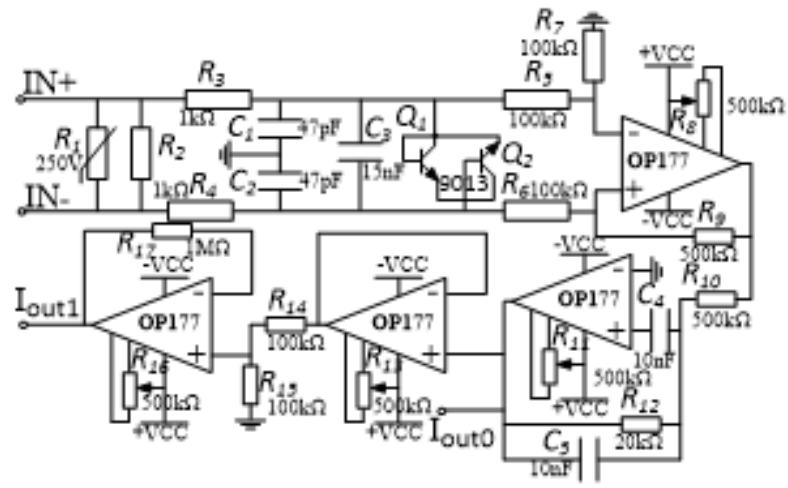

Fig3 Current regulating circuit

Signal through conditioning circuit processing into AD conversion. During AD converting, conversion of synchronicity must be kept, so that the voltage and current signals will not have much deviation in phase, which is advantageous to the grounding resistance calculation. At the same time, choosing the appropriate sampling period can ensure the accuracy of subsequent FFT processing. This article uses the AD7656 to implement parallel synchronous voltage and current signal conversion, while AC synthesis module sends the synchronization control signal to AD conversion to increase the Precision of AD conversion. After converting, data is fed into STM32 for FFT processing, and then calculate the grounding resistance.

\section{Host computer System}

The PC system of ground resistance tester as the interface of human-computer interaction is designed with a reasonable standard. This paper selects ARM11 platform that equips with WinCE system for PC. The PC system can be divided into self-test, ground resistance testing, 485 communication interface, scene description module. When the system is running, it will enter the main interface firstly, and complete system self-test and communication Self-Test. Then it will enter the ground resistance testing interface to measure resistance. Testing at the same time it can manually input field data, draw the diagram of the building, and mark the ground point on the map. It is conducive to the realization of intelligent evaluation, and make data recording more conveniently. Thereby it can replace the way that only using liquid crystal display in previous time, and improve the efficiency of detection.

\section{Software design}

The software module of this detection system mainly realize the human-machine interface, data collection and processing, storage and output function. The software flow chart of the system is shown in figure 4. After the system initialization, detection command will be sent by the PC system, which will control the ac waveform generator work. When the AC synthesis module is stable, STM32 will send the synchronous sampling signal used to open the AD conversion and collect 1024 data points that is used by FFT processing ${ }^{[4]}$. Grounding resistance value can be obtained through the appeal process, and then upload the results to the host computer to save. 


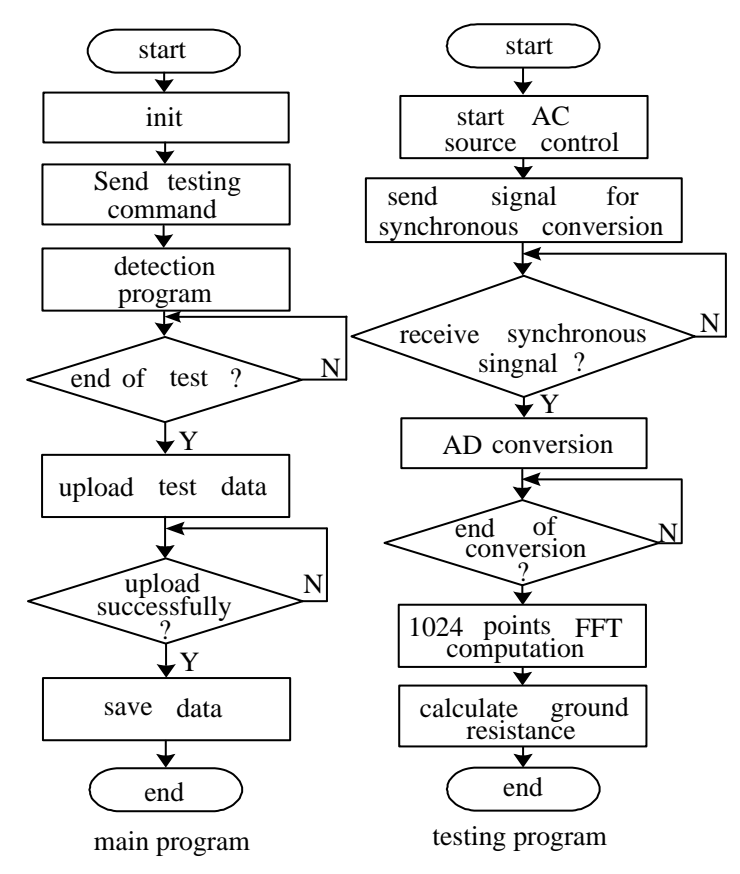

Fig4 Flow chart of Program

\section{Testing and analysis}

Under the experiment condition, this detection system used the standard resistance for the actual test, and compared with commonly used 4105A-type ground resistance detector and analyzed the results, the test results are shown in Table 1. We have used 4105A-type ground resistance detector and the detector that we designed measured in 10 times, so the data in the table were the average of 10 times measurements. From the data in the table, it can be seen that the detector has the characteristics of stability and high precision. In the small resistance measurement, the 4015-type detector can only keep two significant figures, so on the precision, deviation will be relatively large. The detection device can intelligently change the range, which could help improve the detection accuracy. The precision of our detector satisfied the actual needs, and reached the design requirements.

Tab1 Test value comparison table

\begin{tabular}{ccccc}
\hline $\begin{array}{c}\text { Resistance } \\
/ \Omega\end{array}$ & $\begin{array}{c}4015 \text {-type } \\
/ \Omega\end{array}$ & $\begin{array}{c}\text { Detector } \\
/ \Omega\end{array}$ & $\begin{array}{c}4015 \\
\text { deviation/\% }\end{array}$ & $\begin{array}{c}\text { detector devi- } \\
\text { ation } / \%\end{array}$ \\
\hline 0.56 & 0.57 & 0.562 & 1.79 & 0.38 \\
1.25 & 1.24 & 1.24 & 0.81 & 0.81 \\
3.00 & 2.96 & 3.02 & 1.33 & 0.67 \\
5.60 & 5.65 & 5.63 & 0.89 & 0.54 \\
10.50 & 10.56 & 10.45 & 0.38 & 0.48 \\
35.24 & 35.11 & 35.19 & 0.37 & 0.14 \\
\hline
\end{tabular}

\section{Conclusions}

This buildings grounding resistance test system was designed based on three-pole compensation method, at the same the principle of three-pole compensation was introduced detailedly. The composition and function of each module of the system were analyzed. The testing equipment used digital technology to synthesize alternating current, which could ensure proper injected current into ground grid, reduce the size of the instrument and make the testing work more conveniently. The synchronous conversion function of voltage and current signals reduced the measurement error which was bring by the difference of phase between voltage and current. Meanwhile the reasonable signal conditioning circuit and high-speed parallel synchronous AD converter chip were used to effectively eliminate the interference caused by external signals, and improve the detection 
accuracy. The host computer system designed could realize detection, at the same time complete testing data and other relevant data storage, upload, improving the detection efficiency, and ensure tightness and authenticity of the data. The testing instrument with simple operation, high measurement precision, and the advantages of stable performance has certain application value.

\section{Acknowledgment}

The work is supported by General Administration of Quality Supervision, Inspection and Quarantine of China(AQSIQ),No.2013QK023 and the National Natural Science Foundation of Zhejiang Province, No. LY14F040004. The corresponding author is Zhiqiang Chen, email: chenzq@cjlu.edu.cn.

\section{References}

[1] Celal Korasli. Ground resistance measurement with alternative fall-of-potential method[J]. IEEE transactions on power delivery, 2005,20(2):1657-1661.

[2] Cui Jinping, Chen Jiurui, Qu Quan. Lightning protection and grounding resistance measurement error diagnosis[J]. JiLin QIXiang, 2013(4):33-35.

[3] Ding Xiaoping, Lin Guanwen, Yuan Zhihe. Analysis of lighting protection and grounding resistance testing[J]. Modern building electrical, 2012(6):9-11.

[4] Fang Yanmei, Liu Yongqing. Digital signal processing[M]. Beijing: Publishing house of electronics industry, 2007:332-361.

[5] Li Liwei, Zou Jiyan. A new grounding resistance on-line measuring instrument with double jaw[J]. Automation of electric power systems, 2003,27(14):83-87.

[6] Pan Yigang. Analysis of grounding resistance testing[J]. Electrotechincal application, 2009, 28(3):65-69.

[7] Yang Jianming, Pei Sujing, Lu Wei. Importance of grounding resistance during lightning detection and discuss its impact factors[J]. Electronics World, 2014(12):400. 